\title{
Lidar Technique for Measuring Ionospheric Barium Release Ion Density
}

\author{
J. Goree AND J. S. NefF \\ Department of Physics and Astronomy, The University of lowa, lowa City
}

\begin{abstract}
An evaluation of the resonant fluorescence lidar technique as a method of measuring ion density during ionospheric barium releases is presented. We find that it is technically feasible to measure ion densities of $10^{3} \mathrm{~cm}^{-3}$ at an altitude of $200 \mathrm{~km}$ with a time resolution of $0.5 \mathrm{~s}$ by using a ground-based laser and telescope. One application of the lidar technique would be observation of the critical ionization velocity effect at nighttime, without the complication of solar illumination. Criteria useful for evaluating the leasibility of other experiments are presented.
\end{abstract}

\section{INTRODUCTION}

Sounding rocket experiments in the ionosphere are frequently carried out with an explosive canister of barium which detonates, producing a rapidly expanding cloud of metal atoms. To observe these ionospheric releases, we propose a remote sensing method, lidar.

Often, releases are carried out to study the Alfvén critical ionization velocity (CIV) effect [Haerendel, 1982; Wescott et al., 1986a]. As the barium cloud expands, it becomes ionized by solar ultraviolet radiation or, if electrons are heated by waves resulting from a velocity space plasma instability, by electron impact. This latter effect is the CIV phenomenon [Newell, 1985]. A threshold for this condition is that the neutral atom velocity $v$ must exceed $V_{c}$, satisfying $\eta \mathrm{m} V_{c}^{2} / 2=e \Phi_{i}$, where $\eta$ is an efficiency which depends on competing loss mechanisms [Newell and Torbet, 1985] and $e \Phi_{i}$ is the ionization potential of the neutrals. The CIV effect has been claimed to account for the results of laboratory experiments; however, in all but one [Haerendel, 1982] space experiment involving barium releases, the process was not observed.

A series of such tests in space, called the Condor project [Wescott et al., 1986a, b; Kelley et al., 1986; Torbert and Newell, 1986] relied partly upon observation of spontaneous optical fluorescence by cameras located on the ground to determine the ion concentration resulting from the explosion of a shaped charge of an alkaline Earth, such as barium. This method of ion detection has two shortcomings that are corrected with the observation technique proposed here.

The first difficulty is that the release must be performed in the presence of solar radiation, because optical stimulation in the visible is required to produce the fiuorescence. If solar UV radiation is also present, it will ionize the barium. This ionization adds to the ionization due to the CIV effect; thus the method of measurement interfers with the experiment unless the UV component of the sunlight can be blocked. The Star of Lima experimenters attempted to block the UV by launching the barium just before sunrise to an altitude where the visible light would graze through the upper atmosphere but the UV would be absorbed by the ozone [Wescott et al., 1986a]. Although Haerendel [1982] was able to do this in the Porcupine experiment, in the Star of Lima test the rocket overperformed

Copyright 1989 by the American Geophysical Union.

Paper number 88JA03008.

0148-0227/89/88JA-03008\$02.00 and detonated at too high an altitude, so that one could not positively say that UV photoionization did not account for the ion density that the experimenters observed.

The problem of attempting to observe the CIV process in presence of sunlight is quite serious due to the complication of momentum transfer, which Haerendel [1983] explained as follows. In addition to the energy balance that is usually taken into consideration in explaining CIV effect, one must also consider the momentum balance. Ionization of fast neutrals (by any mechanism) produces fast barium ions which lose momentum to the ambient plasma. This causes the rest frame of the ambient plasma to accelerate to a velocity nearer that of the fast barium neutrals. The free energy available to ionize the neutrals through the CIV effect is thus diminished. In the presence of a strong external source of ionization such as photoionization, the CIV process may be entirely suppressed. Thus it is imperative to perform a CIV experiment under conditions where UV photoionization cannot take place. (We should note, however, that Goertz et al. [1985] also studied the momentum coupling process and found this problem to be less severe.)

The second difficulty of relying on passive fluorescence from the ions is that the optical signal represents a column average, along the line of sight, of the ion emissivity. The spatial resolution is thus somewhat limited if only one observation site is used. With several sites better results can be obtained, but only if the weather is fine at each of them and if all the remote cameras function correctly.

\section{Lidar Principles}

The two difficulties outlined above may be avoided by using an active remote sensing technique called lidar. A pulsed laser located on the ground is fired into the ionosphere, where the beam is partly absorbed by the ions. Fluorescence from the ions is then detected by a telescope located on the ground near the laser. The magnitude of the resulting signal yields the ion density and the delay in receiving it yields the range.

When it is used to observe ions in the laboratory, this method is referred to as laser-induced fluorescence (LIF). This laboratory technique is used routinely to monitor barium ions in $Q$ machine plasmas [Hill et al., 1983]. A tunable dye laser is directed through the plasma, and a lens or small telescope collects the ion fluorescence and focuses it though an optical interference filter to a photomultiplier tube (PMT) detector. One thus measures the density of ions that are moving with the velocity that doppler shifts the laser light into resonance 
with the ion's atomic transition. By selecting a laser with a narrow frequency bandwidth and scanning the laser wavelength, the ion velocity distribution function can be measured. Alternatively, by selecting a laser with a large bandwidth so that all ions regardless of their velocity may absorb the laser light, the ion density can be measured.

The equipment and principles of lidar are essentially identical to those of LIF. The principal difference is the distance $R$ between the plasma and the telescope. This difference is important in two ways. First, the larger value of $R$ for lidar allows one to obtain ranging, i.e., spatial resolution. Second, the larger value of $R$ causes the signal, which varies as $1 / R^{2}$, to be much smaller for the ionospheric measurement.

Atmospheric researchers use lidar to observe the Earth's natural sodium layer that is found between the altitudes of 80 and $100 \mathrm{~km}$ [Inaba, 1976; Gardner and Voelz, 1987]. The laser is generally pointed upward at the zenith. The laser light is partially absorbed by the sodium atoms, which have a density of typically $10^{3} \mathrm{~cm}^{-3}$. The fluorescence is collected with a telescope onto a PMT.

A comparison of the sodium layer and a barium release is instructive. The optical transitions for $\mathrm{Na}$ and $\mathrm{Ba}^{+}$are similar. The transition from the ground state of $\mathrm{Na}$ is $3 S_{1 / 2}-3 P_{3 / 2}$, with $\lambda_{0}=589 \mathrm{~nm}$ and an oscillator strength $f=0.65$. For $\mathrm{Ba}^{+}$, the best transition is $6 S_{1 / 2}-6 P_{3 / 2}$, with $\lambda_{0}=455.4 \mathrm{~nm}$ and $f=0.7$. For these two wavelengths, an excimer pumped dye laser has nearly the same efficiency.

The four chief differences in lidar for the sodium layer and a barium release are (1) the barium release is more localized transverse to the laser beam, (2) it has a shorter duration, (3) it has a different velocity distribution, and (4) it is at a higher altitude. Each of these differences will make lidar more difficult for the barium release. The more localized target of gas means that the laser and telescope must be pointed carefully, perhaps with the use of a tracking device to locate the sounding rocket if it is unguided. This means that the rocket would have to carry a beacon. The shorter duration will permit less signal averaging and will require that all the appartus operate properly at the time of the release. The velocity distribution function of the ions must be estimated in advance to obtain a calibrated measurement of their density and to select a laser with an appropriate bandwidth. (This effect is manifested in the parameter $\sigma_{\text {eff }}$, which is discussed later.) Due to its higher altitude, the barium release will result in less signal, according to the inverse square law. If one attempts to counteract the $1 / R^{2}$ effect by exploding the canister at a lower altitude, then the barium ions will collide with atmospheric oxygen atoms, and this will inhibit the CIV effect [Newell and Torbert, 1985].

Despite the four factors mentioned above that make lidar more difficult for the barium release than for the sodium layer, it is still a practical technique. The equipment involved would be virtually identical, except that a larger telescope might be needed. In fact, if one had an existing lidar setup that was designed to probe the sodium layer, the laser apparatus could be adapted inexpensively by changing the laser dye and interference filter and by adjusting the dye laser grating. The signal strength would be adequate for many barium experiments, as shown in the following calculation.

\section{Calculation of Signal Strength}

To predict the optical power $P_{r}$ of the signal that would be received, consider the lidar equation [Inaba, 1976]:

$$
P_{r}=P_{0} A_{r} K^{\prime} T^{2} n(R) \Delta R b \sigma_{\mathrm{eff}} / 4 \pi R^{2}
$$

Here, $P_{0}$ is the laser power, $A_{r}$ represents the telescope area, $K^{\prime}$ is the optical transmission of the apparatus, $T^{2}$ is the twoway atmospheric transmission, $n$ is the number density of the ions at a range $R$, and $\Delta R=c \tau_{l} / 2$. The duration of the laser pulse is $\tau_{l}, b$ is the branching ratio of fluorescence from $6 P_{3 / 2}$ $-6 S_{1 / 2}$, and $\sigma_{\text {efr }}$ is the effective absorption cross section. The result of (1) is used to find the photon count rate

$$
\dot{n}_{d}=\eta P, \lambda_{0} / h c
$$

where $\eta$ is the detector's quantum efficiency.

To estimate $\dot{n}_{d}$, consider the parameters that enter into (1) and (2). All of them except for $\sigma_{\text {eff }}$ can be characterized straightforwardly. The lidar apparatus will be characterized typically by $K^{\prime}=0.2$ and $\eta=0.15$. If a dye laser with $14 \%$ efficiency is pumped by a $500-\mathrm{mJ}$ excimer laser, with pulses $\tau_{l}=17 \mathrm{~ns}$ long, then $P_{0}=4 \mathrm{MW}$. A low-quality $2.5 \mathrm{~m}$ diameter telescope would offer a collecting area of $A_{r}=5 \mathrm{~m}^{2}$. The atmospheric transmission is $T=\exp (K X)$, where $X=1$ at the zenith. The extinction of light due to aerosols and Rayleigh scattering is modeled by $K=A+B \lambda^{-4}$, where the coefficients $A$ and $B$ vary according to geographic location, time of year, and most importantly, weather. Using values typical of Iowa City, $A=-0.08$ and $B=-0.013 \mu \mathrm{m}^{-4}$, we calculate that the transmission at $\lambda=455.4 \mathrm{~nm}$ is $T=0.68$. The twoway transmission through the atmosphere is thus reduced to $T^{2}=0.46$

The effective absorption cross section $\sigma_{\text {eff }}$ depends not only on the atomic absorption cross section, but also on the optical bandwidth of the laser light $\Delta \omega_{l}$ and on the ion velocity distribution function $f_{i}(v)$.

The laser spectral intensity (power versus frequency) $I(\omega)$ will depend on the construction of the dye laser that is used; typically, it will have a Gaussian dependence on $\omega$. We will make the approximation that $I(\omega)$ has a rectangular shape with a constant value $I\left(\omega_{0}\right)$ for $\omega_{0}-\Delta \omega_{l} / 2<\omega<\omega_{0}+$ $\Delta \omega_{1} / 2$ and the value zero otherwise.

To compute an estimate of $\sigma_{\text {eff }}$, consider the laser power absorbed per unit volume, $d W / d t$. In terms of the cross section $\sigma_{\mathrm{efr}}, d W / d t=\hbar \omega n_{p h} n_{i} \sigma_{\mathrm{eff}} c$, where $n_{p h}$ and $n_{i}$ are the number densities of photons and ions, and $c$ is the speed of light. The photon density is eliminated by considering that $I\left(\omega_{0}\right) \Delta \omega_{l}=$ $\hbar \omega n_{p h} c$. This yields

$$
d W / d t=I\left(\omega_{0}\right) \Delta \omega_{l} n_{l} \sigma_{\mathrm{efr}}
$$

Here, the laser light power per unit area per unit frequency is $I(\omega)$, and $\omega_{0}=2 \pi c / \lambda_{0}$ is the atomic transition resonance frequency.

Now, from a semiclassical atomic physics model [Demtroder, 1982], the absorbed power density is also

$$
d W / d t=\int_{0}^{\infty} \alpha(\omega) I(\omega) d \omega
$$

neglecting doppler broadening. The absorption coefficient $\alpha$ is modeled by a Lorentzian,

$$
\alpha(\omega)=\frac{e^{2}}{2 \varepsilon_{0} m_{e}} \frac{n_{i} \pi}{\lambda \omega_{0}} \frac{f \gamma}{\left(\omega-\omega_{0}\right)^{2}+(\gamma / 2)^{2}}
$$

where $f$ is the oscillator strength.

To take into account doppler effects, $\omega$ is replaced by $\omega-k v$ on the right-hand side in (5), and (4) is generalized to

$$
d W / d t=\int_{-\infty}^{+\infty} \int_{0}^{\infty} \alpha(\omega-k v) I(\omega) f_{i}(v) d \omega d v
$$


TABLE 1. Parameters Used in Calculating Photon Count Rate

\begin{tabular}{cl}
\hline Parameter & \multicolumn{1}{c}{ Value } \\
\hline$\lambda_{0}$ & $455.4 \mathrm{~nm}$ \\
$P_{0}$ & $4 \mathrm{MW}$ \\
$A_{r}$ & $5 \mathrm{~m}^{2}$ \\
$K^{\prime}$ & 0.2 \\
$\eta$ & 0.15 \\
$T^{2}$ & 0.46 \\
$n_{i}$ & $10^{3} \mathrm{~cm}^{-3}$ \\
$\tau_{l}$ & $17 \mathrm{~ns}^{2}$ \\
$b$ & 0.74 \\
$R$ & $200 \mathrm{~km}$ \\
$\Delta_{v}$ & $6 \mathrm{~km} / \mathrm{s}$ \\
$N$ & $50 \mathrm{pulses}$ \\
\hline
\end{tabular}

Assume now that the barium release results in an ion velocity distribution function characterized by a width $\Delta v$, which corresponds to a doppler width $\Delta \omega_{d}=2 \pi \Delta v / \lambda_{0}$. For example, if $\Delta v=6 \mathrm{~km} / \mathrm{s}$, then $\Delta \omega_{d}=8 \times 10^{10} \mathrm{rad} / \mathrm{s}$. If the experimenter knows in advance the value of $\Delta v$, then a laser grating can be chosen so that $\Delta \omega_{l} \approx \Delta \omega_{d}$ to yield the largest possible lidar signal. To model the distribution function, a rectangular shape matching that of $I(\omega)$ can be used. Doing this, and approximating $\alpha(\omega-k v)$ as a delta function (which is appropriate because $\omega_{0} \gg \Delta \omega_{l} \gg \gamma$ ), then (6) becomes

$$
d W / d t=I\left(\omega_{0}\right) n_{l} f \frac{\pi e^{2}}{2 c \varepsilon_{0} m_{e}}
$$

Combining (3) and (7) yields

$$
\sigma_{\text {eff }}=f \frac{\pi e^{2}}{2 c \varepsilon_{0} m_{e}} \frac{1}{\Delta \omega_{l}}
$$

Using the choice $\Delta \omega_{1} \approx \Delta \omega_{d}$, the estimate of $\sigma_{\text {eff }}$ in (8) becomes

$$
\sigma_{\text {eff }}=f \frac{\lambda_{0} e^{2}}{4 c \varepsilon_{0} m_{e}} \frac{1}{\Delta v}
$$

The result expressed by (9) assumes that the experimenter has tuned the laser light frequency to the resonant frequency of the ion $\omega_{0}$, corrected by the doppler shift due to the velocity of the rocket and the velocity of the gas from the exploding shaped charge. The approximations made in deriving (9), especially the models chosen for $f_{i}(v)$ and $I(\omega)$, will make our result different from that of an actual specific experiment. The experimenter can use the expressions presented above and enter the specific parameters that apply to his or her experiment. With this caveat in mind, let us assume $\Delta v=6 \mathrm{~km} / \mathrm{s}$ in a barium release. This yields an effective absorption cross section of $\sigma_{\text {eff }}=1.4 \times 10^{-16} \mathrm{~m}^{2}$.

Using the above result in (1) and (2), with the parameters that have been presented above and also in Table 1 , the photon count rate $\dot{n}_{d}$ will be $3.3 \times 10^{5} \mathrm{~s}^{-1}$. This is our signal.

\section{Signal-To-Noise Ratio}

To show that this signal is adequate, we must do two things. First, we should compare it to the count rate for the noise to find an estimate of the steady state signal-to-noise ratio (SNR). Second, we must consider that the signal is integrated over a limited duration of time; therefore only a finite number of photons will be collected, and the statistical fluctuations in this quantity must be considered. The latter effect will turn out to be the dominant source of noise and will thus determine the overall SNR.
The signal is averaged over a finite amount of time, and a finite number of photons are thus counted. The PMT signal is registered on a device called a range-gated photon counter. In this instrument, photons are counted in several sequential bins. The delay of the bin with respect to the time the laser fires corresponds to the range. The width of the bin determines the range resolution. For example, if the bin width is $10 \mu \mathrm{s}$, the range resolution will be $1.5 \mathrm{~km}$. For the count rate $\dot{n}_{d}=$ $3.3 \times 10^{5} \mathrm{~s}^{-1}$ that was found above, an average of 3 photons would be counted in each bin for a single laser pulse. The single pulse SNR due to the photon statistics would thus be approximately 2 .

For comparison, consider the steady state SNR. It is determined by two sources of noise: skylight and detector shot noise. The telescope must be adjusted to view only the area of the sky illuminated by the laser, and not any more, in order to minimize this effect. We will assume that the telescope receives a solid angle corresponding to a 1-km diameter spot at range of $200 \mathrm{~km}$. Also, we assume the interference filter bandwidth is $0.5 \mathrm{~nm}$, and the parameters listed in Table 1, including the system transmission $K^{\prime}$ and detector efficiency $\eta$. At zenith at nighttime the skylight noise will then be $10^{3} \mathrm{~s}^{-1}$. The detector shot noise will be only $10 \mathrm{~s}^{-1}$ if the PMT is cooled. The skylight noise is the greater of two. Using its value, we find that the steady state SNR will be approximately 330 , which is greater than the steady state SNR found above. Photon statistics, and not skylight or detector noise, will thus dominate the noise entering into the overall SNR.

The statistics are improved by averaging over multiple laser pulses. This is done at the expense of range and temporal resolution. To weigh the benefits of signal averaging sensibly, we must consider the resolution that would be required to observe the barium release. Since the CIV process occurs on a time scale of approximately $10 \mathrm{~s}$, a temporal resolution of $0.5 \mathrm{~s}$ would offer adequate observation of the development of the release. An excimer-pumped dye laser can fire 100 pulses/s. One would thus average over 50 pulses and count an average of 165 photons per bin. The corresponding statistical noise of $(165)^{1 / 2}$ photons would yield an overall SNR of 13 . In other words, the error bars in the measurement of $n_{i}$ would be $8 \%$. This noise level should be acceptable for a CIV experiment.

In addition to the random errors analyzed above, systematic errors must be considered in fielding the lidar apparatus. Two factors which could be dealt with successfully, exactly as one does with sodium layer lidar, are the value of laser power $P_{0}$, which can be measured accurately with a calorimeter, and the detector system efficiency $\eta K^{\prime}$, which can be calibrated at the site by Rayleigh or Raman scattering on the atmosphere. A greater problem may lie in the estimate of the ion velocity distribution function, which enters into the calculation of $\sigma_{\text {eff }}$. This must be known in advance of the experiment in order to set up the laser and to determine the absolute magnitude of the ion density from the data. A distribution function can be produced by numerical particle simulation if experimental data is not available. Provided that this estimate can be made accurately, then systematic errors may be less than the random errors. In that case, the ion density measurement can be made with an accuracy near the $8 \%$ level found above. If a different set of parameters applies to an experiment, then the criteria presented above can be used to compute the signal strength and the size of the error bars.

\section{EXPerimental Configuration}

The most likely configuration that the experimenter would choose would have both the laser and the telescope located on 
the ground. The signal strength and noise levels presented in the last two sections were calculated for this arrangement. One might also consider using a smaller telescope and installing it in an instrumentation package that is flown on the same sounding rocket as the barium charge. The laser, however, must be located on the ground because the weight and electrical power consumption are both much to large for it to be put aboard a sounding rocket. (The only space vehicle likely to offer enough room and electrical power for an excimer-pumped dye laser would be NASA's proposed space station.)

The experiment must be performed at high to mid-latitudes. This requirement is due to the importance of the Earth's magnetic field. As explained above, the distribution function must be well-characterized to set up the laser and to interpret the data. This distribution function is the projection of the threedimensional distribution function onto the direction of the laser beam. In a CIV experiment, the distribution function perpendicular to the Earth's magnetic field will change during the course of the release, as can be seen in the phase-space plots of Machida and Goertz [1986]. In the direction parallel to the magnetic field, on the other hand, the distribution function will not vary as much and will be better characterized in advance of the experiment. The experimenter would choose therefore to point the laser beam parallel to the magnetic field. This would require that the experiment not be performed at low latitudes.

The CIV process will not occur readily unless the neutral atoms are injected with a nonzero velocity component perpendicular to the magnetic field. Therefore the shaped charge of barium should not be oriented to fire parallel to the laser beam, but rather obliquely to it.

The data produced by the lidar technique will be a plot of ion density versus range or altitude. This plot would be a snapshot of the density profile along the laser beam at a given time, with a new snapshot produced every half second. One could choose either to leave the direction of the laser beam the same during the entire release or to repoint it every half second to cover a raster in the sky. It might be desirable to do the latter if one expected an ionization front to sweep across the sky transverse to the laser beam and wished to observe this spatial effect.

\section{CONCLUSIONS}

We have shown that the lidar apparatus of the type already in use for studying the atmospheric sodium layer can be easily adapted to observe barium ions in a shaped charge release at nighttime. This would avoid the use of sunlight to see the ions in critical velocity experiments. Adequate signals can be attained if $n_{i}=10^{3} \mathrm{~cm}^{-3}$ and the range is $200 \mathrm{~km}$. Using this method would produce plots of the ion density versus range, and these plots would be updated at intervals of $0.5 \mathrm{~s}$. The parameters presented here result in $8 \%$ error bars in a measurement of ion density with range resolution of $1.5 \mathrm{~km}$. We also present criteria useful for an experimenter to predict the signal and noise levels for experiments having other parameters.

Acknowledgments. We thank G. Haerendel for a critical reading of the manuscript, and C. K. Goertz, P. Kleiber, and W. Stwalley for helpful discussions. This work was supported in part by an IBM Faculty Development Award. J. Goree is supported in part by the NORAND Applied Academics Program.

The Editor thanks C. S. Gardner and R. B. Torbert for their assistance in evaluating this paper.

\section{REFERENCES}

Demtroder, W., Laser Spectroscopy, Springer-Verlag, New York, 1982.

Gardner, C. S., and D. Voelz, Lidar studies of the nighttime sodium layer over Urbana Illinois, J. Geophys. Res., 92, 4673, 1987.

Goertz, C. K., S. Machida, and R. A. Smith, An asymptotic state of the critical ionization velocity phenomenon, J. Geophys. Res., 90, $230,1985$.

Haerendel, G., Alfvén's critical velocity experiment tested in space, $Z$. Naturforsch, A37, 728, 1982.

Haerendel, G., The role of momentum transfer to the ambient plasma in critical velocity experiments, Active Experiments in Space Symposium, Eur. Space Agency Spec. Publ., 245, 1983.

Hill, D. N., S. Fornaca, and M. G. Wickham, Single frequency scanning laser as a plasma diagnostic, Rev. Sci. Instrum., 54, 309, 1983.

Inaba, H., Detection of atoms and molecules by raman scattering and resonance fluorescence, in Laser Monitoring of the Atmosphere, edited by E. D. Hinckley, Springer, New York, 1976.

Kelley, M. C., R. F. Pfaff, and G. Haerendel, Electric field measurements during the condor critical velocity experiment, J. Geophys. Res., 91, 9939, 1986.

Machida, S., and C. K. Goertz, A simulation study of the critical ionization velocity process, J. Geophys. Res., 91, 965, 1986.

Newell, P. T., Review of the critical ionization velocity effect in space, Rev. Geophys., 23, 93, 1985.

Newell, P. T., and R. B. Torbert, Competing atomic processes in $\mathrm{Ba}$ and $\mathrm{Sr}$ injection critical velocity experiments, Geophys. Res. Lett., $12,835,1985$.

Torbert, R. B., and Patrick T. Newell, A magnetospheric critical velocity experiment: Particle results, J. Geophys. Res., 91, 9947, 1986.

Wescott et al., E. M., Star of lima: Overview and optical diagnostics of a barium Alfvén critical velocity experiment, J. Geophys. Res., 91, 9923, 1986a.

Wescott et al., E. M., Star of condor: A strontium critical velocity experiment, Peru 1983, J. Geophys. Res., 91, 9933, 19866.

J. Goree and J. S. Neff, Department of Physics and Astronomy, The University of Iowa, Iowa City, IA 52242.

(Received April 7, 1988; revised June 2, 1988; accepted June 6, 1988.) 